\title{
Pedagogía de la confianza: una estrategia para generar ambientes escolares de paz
}

\author{
Ninfa Pinilla Quiñones* \\ María Del Carmen Mendieta González ${ }^{* * *}$
}

Recibido: 7 de febrero de 2017 - Aprobado:28 de abril de 2017

\section{Resumen}

La convivencia escolar es un tema recurrente desde las últimas décadas del siglo XX y comienzos del siglo XXI, en razón de los altos índices de violencia y su repercusión negativa en el aprendizaje y la construcción de habilidades sociales y comunicativas de los estudiantes. Por ello, en la institución educativa Liceo Nacional José Joaquín Casas, sede General Santander, de Chiquinquirá, entre los años 2011 a 2013 se desarrolló el proyecto Pedagogía de la Confianza, orientado al manejo y posible solución de los constantes conflictos que se presentaban entre los niños y las niñas de los grados tercero, cuarto y quinto, muchas veces resueltos de forma violenta. Dicha experiencia, abordada como un estudio de caso, fue sistematizada para: 1) dar cuenta de la praxis pedagógica en contextos locales, que en muchas ocasiones no es reconocida por la comunidad ni por el Estado; 2) identificar y comprender las acciones que aportan en la resolución pacífica de conflictos, así como en la cimentación de una cultura del buen trato, y 3) visibilizar el uso de narrativas aplicadas con estudiantes, docentes y directivos de la institución, con resultados satisfactorios.

Palabras clave: convivencia escolar; violencia escolar; resolución de conflictos; buen trato; pedagogía de la confianza

Artículo de investigación. Este trabajo fue realizado en el marco de la Maestría de Derechos Humanos de la Universidad Pedagógica y Tecnológica de Colombia - seccional Chiquinquirá. DOI: http://dx.doi.org/10.15332/ s0120-8454.2017.0091.03

** Estudiante de Maestría en Derechos Humanos, UPTC sede Chiquinquirá. Especialista en Gerencia y Proyección Social de la Educación. Coordinadora en la Escuela Normal Superior de Saboyá, Colombia. Correo electrónico: ninfapinilla@ yahoo.es. ORCID: orcid.org/0000-0002-6664-7179

*** Estudiante de Maestría en Derechos Humanos, UPTC sede Chiquinquirá, Colombia Licenciada en Español y Literatura. Docente de básica secundaria. Correo electrónico: mendietams@hotmail.com. ORCID: http://orcid. org/0000-0003-1036-4521 


\title{
Pedagogy of trust: a strategy to generate peace environments at school*
}

\author{
Ninfa Pinilla Quiñones** \\ María Del Carmen Mendieta González ${ }^{* * *}$
}

\section{A bstract}

School coexistence has been a recurring theme since the last decades of the twentieth century and the beginning of the twenty-first century, due to the high rates of violence and its negative impact on learning and the building of social and communicative skills of students. Therefore, in the Liceo Nacional Jose Joaquín Casas school, General Santander headquarters in Chiquinquirá was developed the Pedagogy of confidence project during the years 2011 and 2013. It was developed in order to give a possible solution of the constant conflicts that were presented among third, fourth and fifth graders. This experience as a case study was systematized to: 1) show pedagogical praxis in regional contexts, which in many cases is not recognized by the community or the government; 2) identify and understand the actions that support the peaceful resolution of conflicts, as well as in the foundation of a culture of good treatment; And 3) make visible the use of narratives applied to students, teachers and executives of the institution, with satisfactory results.

Keywords: school coexistence; school violence; conflict resolution, good treatment;, Pedagogy of confidence

Research article. This work was made within the framework of the Master's Degree in Human Rights of the Pedagogical and Technological University of Colombia - Chiquinquirá section. DOI: http://dx.doi.org/10.15332/s0120-8454.2017.0091.03

** Master's Degree in Human Rights student, UPTC Chiquinquirá branch. Specialist in Management and Social Projection of Education. Coordinator at the Higher School of Saboyá, Colombia. Email: ninfapinilla@yahoo.es. ORCID: orcid. org/0000-0002-6664-7179

*** Master's Degree in Human Rights student, UPTC, Chiquinquirá branch, Colombia Graduate in Spanish and Literature Teacher of basic secondary. Email: mendietams@hotmail.com. ORCID: http://orcid.org/0000-0003-1036-4521 


\title{
Pédagogie de la confiance: une stratégie pour créer des espaces scolaires en paix
}

\author{
Ninfa Pinilla Quiñones* \\ María Del Carmen Mendieta González
}

\section{Résumé}

La vie scolaire est un thème récurrent depuis les dernières décennies du XXe siècle et au début du XXIe siècle, en raison des taux élevés de violence et de son impact négatif sur l'apprentissage et l'acquisition de compétences sociales et de communication des étudiants. C'est pour cela, que l>institution éducative Lycée National José Joaquín Casas, General Santander, de Chiquinquirá (Colombie), a développé entre 2011 et 2013, le projet Pédagogie de la Confiance, orientée à la gestion et aux possibles solutions aux conflits permanents qui se présentaient entres les garcons et les filles du cycle III, IV et V, qui étaient très souvent résolus de façon violente. Cette expérience, abordée comme une étude de cas, fût systématisée pour : 1) rendre compte de la praxis pédagogique dans les contextes locaux, car très souvent elle n'est pas reconnue par la communauté, ni par l'État ; 2) identifier et comprendre les actions qui permettent une solution pacifique des conflits, ainsi que dans la création d'une culture de respect, et 3) montrer l'usage de récits appliqués avec les étudiants, les professeurs et le comité directeur de l'insititution, avec des résultats satisfaisants.

Mots clés: vie scolaire; violence scolaire; résolution de conflits; respect; pédagogie de la confiance

Article de recherche. Ce travail a été réalisé dans le cadre de la Maîtrise en Droits de l'Homme de l'Université Pédagogique et Technologique de Colombie - section Chiquinquirá. DOI: http://dx.doi.org/10.15332/s0120-8454.2017.0091.03

** Étudiante de la Maîtrise en Droits de l'Homme, UPTC, section Chiquinquirá, Colombie. Spécialiste en Gestion et Projection Sociale de l'Éducation. Coordinatrice à l'École Normale Supérieure de Saboyá.Email: ninfapinilla@yahoo.es. ORCID: orcid.org/0000-0002-6664-7179

*** Étudiante de la Maîtrise en Droits de l'Homme, UPTC, section Chiquinquirá, Colombie. Licence en Espagnol et Littérature. Professeur au secondaire. Email: mendietams@hotmail.com. ORCID: http://orcid.org/0000-0003-1036-4521 


\section{Introducción}

Según el informe de la Subsecretaría de Asuntos para la Convivencia y Seguridad Ciudadana, "el pleno desarrollo del potencial humano de quienes se encuentran en proceso de formación depende tanto de la calidad de la experiencia cognoscitiva como del ambiente de convivencia y seguridad que brinden los centros educativos a sus estudiantes" (2006, p. 17). De acuerdo con este planteamiento, los colegios deben brindar a sus estudiantes ambientes agradables que les permitan desarrollar sus potencialidades y establecer relaciones armónicas con sus compañeros.

Los ambientes escolares en 885 escuelas de Bogotá y sus alrededores atraviesan desde hace varios años condiciones adversas, como se refleja en un estudio realizado con 87.207 estudiantes en el año 2008, donde se indica que "cerca de uno de cada tres reporta haber sufrido agresión física en el último mes, cerca de tres de cada diez admiten haber agredido físicamente a compañeros en el último mes" (Chaux, 2012, p.32), datos que hacen visible la problemática de violencia escolar. De otra parte, una encuesta realizada por el Departamento Administrativo Nacional de Estadística (DANE, 2011) en el marco de un acuerdo de cooperación con la Secretaría de Educación de Bogotá, preguntó por la existencia de un ambiente agradable y de sana convivencia en el salón de clase, a lo cual los estudiantes de grado quinto respondieron en un $40.5 \%$ de manera negativa. A su vez, en una investigación realizada en Bogotá y cinco municipios de Cundinamarca por Carvajal, Urrea y Soto (2012, p. 59), los estudiantes manifestaron que el clima escolar es poco satisfactorio. Lo anterior refleja que al interior de las instituciones educativas no solo existen problemáticas que alteran la sana convivencia entre los educandos, sino donde la violencia escolar pasa a un primer plano requiriendo alternativas de cambio estructurales que permeen la comunidad educativa.

Encontramos importante, entonces, indagar sobre experiencias pedagógicas que se vienen llevando a cabo para contrarrestar la violencia escolar y, a través de su sistematización, rescatar saberes y prácticas que cotidianamente realizan los docentes y en general la comunidad educativa para resolver los problemas de este tipo que se presentan en su contexto educativo. Ese es el objetivo de este estudio realizado en la institución educativa Liceo Nacional José Joaquín Casas, sede General Santander de Chiquinquirá, con estudiantes de los grados tercero, cuarto y quinto durante los años 2011, 2012 y 2013, denominado Pedagogía de la Confianza, mediante la aplicación de encuestas, entrevistas semiestructuradas, revisión de documentos y el uso de narrativas.

Inicialmente se presentan algunos antecedentes para visibilizar las problemáticas de violencia escolar y conflictos que se viven en algunas instituciones educativas de varios municipios del país como: Institución Educativa (I. E.) Julián Pinto Buendía de Cereté, I. E. Escuela Normal Superior de Saboyá, I. E. Gustavo Romero Hernández de Tibaná y nueve instituciones educativas de cinco 
municipios (Cogua, Sopó, Tenjo, Tocancipá y Zipaquirá) del departamento de Cundinamarca; acto seguido, se expone la metodología, donde se da a conocer el enfoque y los instrumentos utilizados para la recolección de la información, se caracteriza el municipio y la población con la cual se realizó la experiencia; en un tercer aparte se describe la experiencia y se sustentan dos categorías teóricas asociadas a la investigación: conflicto escolar y convivencia pacífica; finalmente se dan a conocer los resultados y las conclusiones.

\section{Conflicto escolar y convivencia pacífica:}

\section{una perspectiva teórica}

Para comprender el sentido y significación del conflicto en el contexto sociocultural de las comunidades humanas, tomamos en cuenta la definición aportada por Girard y Koch (1997): “El conflicto es una dinámica interpersonal o intergrupal que refleja contradicciones y controversias que, bien manejadas, generan procesos constructivos basados en la buena comunicación" (p. 33). Punto de vista que encierra el accionar de las personas como seres sociales que pertenecemos a una determinada comunidad, donde se generan oposiciones y desacuerdos que comúnmente se denominan conflicto.

Para Morton Deutsch (2003), "el conflicto existe cuando ocurren actividades incompatibles entre las personas" (p. 5), y Rosa Alzate (1998) afirma que el conflicto se asocia a "la incompatibilidad de conductas, cogniciones (incluyendo las metas) y/o afectos entre grupos o individuos que pueden conducir o no a una expresión agresiva de su incompatibilidad social" (p. 17). Estas son dos definiciones formales que coinciden en expresar que el conflicto existe dentro de las comunidades o grupos de personas, quienes por sus diferencias no llegan a acuerdos, según sus pretensiones. En otras palabras, el conflicto es inherente o característico de las personas que coexisten con otros. Sin embargo, lo malo no es que exista el conflicto, sino que no se sepa cómo solucionarlo sin que se convierta en un hecho violento.

Para Lederach (1997), el conflicto se presenta en todas las culturas y en todos los tiempos de la historia de la humanidad, y los problemas que surgen de estos se deben concebir "como algo que demanda una solución, y no como una batalla que se debe ganar" (p. 13). Por tanto, se requiere que el conflicto genere un espacio para el diálogo, la discusión de puntos de vista de intereses, la tolerancia y el respeto a la dignidad humana.

La mediación es una forma o estrategia para abordar los conflictos; es una herramienta que conlleva valores y procedimientos que educan en la paz y consolidan una actuación profundamente democrática. La mediación “es un proceso cooperativo de resolución de conflictos, en donde la idea es que las partes encuentren una solución a la disputa" (Girard y Koch, 1997, p. 46), lo cual implica construir un sentido más fuerte de cooperación y comunidad con 
el grupo, mejorar el ambiente por medio de la disminución de la tensión y la hostilidad; desarrollar el pensamiento crítico y las habilidades en la solución de problemas; mejorar las relaciones entre las personas; desarrollar las habilidades para el liderazgo positivo, y facilitar la comunicación y las habilidades para la vida cotidiana.

Para Acosta (2004), "la mediación es un tipo de negociación en que las partes en conflicto siguen las sugerencias de un tercero durante el proceso de establecimiento de acuerdos" (p. 216). De esta manera, resulta importante que una persona neutral escuche a las partes implicadas en el conflicto para así encontrar soluciones por vías del dialogo y mediante formas no violentas. Recurriendo a sistemas de mediación, las partes se sienten satisfechas por el acuerdo convenido y esta situación las predispone favorablemente para abordar futuros conflictos de la misma manera. La mediación busca la negociación integradora, se basa en el consenso, la colaboración y una actitud constructiva hacia la búsqueda de la convivencia en paz.

Las anteriores maneras de accionar frente al conflicto bien pueden aplicarse en el contexto cotidiano de las personas en la escuela, por ser en este plano donde se debe trabajar para mejorar la convivencia entre pares, entre estudiantes y docentes, quienes comparten gran parte de su tiempo en este escenario y, por lo mismo, generan determinadas disyuntivas que se deben solucionar en aras de la convivencia en armonía.

De otro lado está el propósito de la convivencia pacífica, entendida como la base de la democracia. Aplica en todos los niveles de la vida, desde la relación entre padres e hijos (en la familia) hasta la relación en lo laboral, con la naturaleza, el campo, la ciudad y con grupos de la comunidad. La convivencia pacífica es algo que se debe poner en práctica todos los días para tener ambientes amables y menos hostiles. De esta manera, las personas se sentirán a gusto en su comunidad y podrán realizarse mejor en todos los aspectos. Según Huertas y Montero (2001), la convivencia es:

Ayudar, colaborar con una acción conjunta, es congregarse para un fin determinado, buscando el bienestar de todos; es vivir en compañía de otros para compartir, en busca de uno, de los otros y de la naturaleza. Cuando el individuo es el responsable de su vida y también de la vida y el destino de otros, del rumbo de la sociedad a la cual pertenece, de la conservación y protección del medio ambiente, deben conocer, antes que nada, sus aptitudes con el fin de ponerlas al servicio y bienestar de todos. Como no está solo, ni crece solo, sino de manera permanente se relaciona y convive, encuentra diversos espacios para la convivencia y la socialización. (p. 65)

En la cita antes referenciada se destaca la convivencia como algo inherente a los seres humanos, quienes necesitan vivir en grupo como condición propia de su ser social; de ahí que la familia, la escuela, el barrio, la comunidad sean 
agrupaciones en las cuales los niños desde muy temprano deben aprender a vivir. Como dice Zurbano (2008):

La paz o convivencia pacífica afecta a toda persona. Es un proceso complejo que incide en todos los ámbitos de la vida, de modo que el individuo se siente y actúa en armonía consigo mismo, con los demás y con el medio ambiente. Armonía es sinónimo de orden, de equilibrio, de sentido justo de la realidad. Educar para la paz es desarrollar todos aquellos valores que son necesarios para el desarrollo armónico de la personalidad. La paz aglutina los valores fundamentales para la vida. (p. 22)

Esta afirmación da relevancia a la generación de ambientes escolares de paz y, a partir de este aprendizaje, de la convivencia en paz como condición necesaria para la vida dentro del grupo o grupos a los cuales se pertenece. Sin embargo, para la realización de dichos propósitos se hace indispensable la construcción de un ambiente de confianza que favorezca e incentive la participación colectiva en el diálogo y la resolución de problemas. Es por ello que cobra relevancia la pedagogía de la confianza, por su práctica "orientada a que los estudiantes cultiven la confianza en sus propias capacidades; en sus profesores; [...] en el grupo de compañeros y compañeras; en el personal directivo de la escuela; en el entorno comunitario, y en las instituciones sociales" (Hevia, 2016, p. 38). De esta forma se potencializan las aptitudes de niños y niñas, se cimientan relaciones interpersonales, se aprende el autocuidado y el cuidado por los otros.

La pedagogía de la confianza aplica varios mecanismos para reforzar la generación de relaciones de confianza en su interior, entre los que se destaca: la generación de normas de comportamiento y cooperación consensuadas, el ejercicio de una autoridad responsable, la socialización en valores y participación (Hevia, 2006, p. 75). Mecanismos que llevados a la práctica permiten una concepción positiva de las relaciones interpersonales, la prevención del maltrato y comunidades que establecen una comunicación más armónica. Dicha estrategia trabajada con los estudiantes es un aporte institucional para la convivencia pacífica.

\section{A modo de antecedentes}

El término clima escolar en nuestro país comienza a cobrar importancia en las dos últimas décadas del siglo XX: "en Colombia, la experiencia sobre el problema de violencia escolar, asociada a la connotación de clima escolar, se inicia en la década de los años 80, con los trabajos de Parra, S., Araceli de Tesanos y Camargo, M." (Erazo, 2010, citado en Peñaranda, 2006, p. 10). Sin embargo, los estudios sobre clima escolar en el país son poco comunes.

Para el año 2008, el Ministerio de Educación Nacional (MEN) se ocupa del clima escolar en la Guía para el mejoramiento institucional de la autoevaluación al Plan de 
Mejoramiento, en donde lo define como un proceso de la gestión directiva encaminado a "generar un ambiente sano y agradable que propicie el desarrollo de los estudiantes, así como los aprendizajes y la convivencia entre todos los integrantes de la institución" (p. 28). Entiende el MEM, de esta manera, que para el desarrollo de los aprendizajes y habilidades personales y sociales de los estudiantes se hace necesario un ambiente escolar agradable. Un componente importante del clima escolar, como se menciona en la misma guía, es la convivencia escolar, que debe permitir a todos los miembros de la comunidad el relacionarse con los otros de manera armónica y asertiva.

La convivencia escolar cuenta con un amplio número de estudios centrados principalmente en el análisis de la violencia escolar, temática que se puede considerar reiterativa en especial en sus discusiones y conclusiones sobre las manifestaciones, lugares de ocurrencia e incidencia de los entornos, entre otros aspectos del problema. En cuanto a las expresiones de violencia entre estudiantes, se encuentran las agresiones físicas por medio de empujones, patadas, cachetadas y puños; agresiones verbales como insultos, apodos, frases ofensivas; acciones de exclusión, y daño de materiales o de útiles escolares (Ramírez y Arcila, 2013, p. 420; Patiño, 2011, p. 88). Tales acciones se consideran una demostración evidente de la intimidación, la coacción y el temor que producen, y se expresan como la realidad de la violencia que se genera en el entorno escolar.

Entre los lugares de ocurrencia de dichas agresiones se encuentra en primer lugar el aula de clase, seguido por el patio de recreo y en menor proporción en sitios como las salidas de los baños y la tienda escolar (Carvajal, Soto y Urrea, 2012; Hernández y Suárez, 2013).Resulta preocupante que el aula de clase, donde se desarrolla la mayor parte de la labor pedagógica, se convierta en un sitio inseguro para los educandos, quebrantando su significación como el lugar privilegiado donde se socializan conocimientos, interactúan las personas, se vivencian los valores y se agencian los saberes. Pero, lastimosamente, los entornos externos a la escuela ejercen una influencia negativa en los comportamientos de las y los estudiantes. Idea que se refuerza con el criterio de Chaux (2012) cuando señala:

El aula es el entorno en el que cotidianamente viven y se relacionan los niños y niñas; puede influir en su forma de ser y de actuar, según sea si ellos están expuestos a episodios constantes de violencias y agresiones en la familia, la escuela y/o la comunidad se puede convertir en un refuerzo de comportamientos agresivos. De tal manera, que se corre el riesgo que las violencias vividas u observadas se repliquen en sus actuaciones diarias en los escenarios que comparten con otros, entre ellos en el ambiente escolar. (p. 44)

De hecho, la escuela se ha convertido en uno de los escenarios donde se reproduce la violencia entre pares. Frente a esta problemática generalizada, en Colombia se ha legislado en pro de la promoción de la convivencia pacífica escolar y la mitigación de la violencia, como lo demuestra, por ejemplo, la promulgación 
de la Ley General de Educación (Ley 115 de 1994), que en su artículo 5, numeral 2 , señala como uno de los fines de la educación "la formación en el respeto a la vida y a los demás derechos humanos, a la paz, a los principios democráticos; de convivencia, pluralismo, justicia, solidaridad y equidad, así como en el ejercicio de la tolerancia y la libertad". Obsérvese que desde el año 1994 se hace referencia a que la educación debe orientarse a la formación de sujetos capaces de vivir en paz, con habilidades que les permitan relacionarse con los demás y con el entorno.

En el año 2004, el MEN publica los Estándares básicos de competencias ciudadanas, orientados, entre otros, a "lograr relaciones más armoniosas en las instituciones educativas" (p.148). Estos se encuentran divididos en tres grandes grupos, uno de ellos el de convivencia y paz, que busca "formar a los y las estudiantes para que cuenten con las competencias y conocimientos necesarios para relacionarse con otras personas de manera pacífica y constructiva, sin recurrir a la violencia" (p. 149).

Nueve años más tarde se promulga la Ley 1620 de 2013, creativa del "Sistema Nacional de convivencia escolar y formación para el ejercicio de los derechos humanos, la educación para la sexualidad y la prevención y mitigación de la violencia escolar", que en el artículo 4 numeral 7 destaca uno de los objetivos del sistema: "orientar estrategias y programas de comunicación para la movilización social, relacionadas con la convivencia escolar, la construcción de ciudadanía y la promoción de los derechos humanos, sexuales y reproductivos". Esta ley establece un mayor compromiso de parte de todos los miembros de la comunidad educativa (estudiantes, educadores, padres de familia, egresados, directivos docentes y administradores escolares) para promover una mejor convivencia de los niños, niñas y adolescentes de los establecimientos educativos del país, que permita prevenir y mitigar la violencia escolar.

\section{Acercándonos al territorio de Chiquinquirá}

El departamento de Boyacá no ha sido ajeno a las violencias que ha padecido el país a lo largo del siglo XX e inicios del siglo XXI, como la violencia bipartidista con su principal auge en la década de los cincuenta, la presencia de grupos armados al margen de la ley y la guerra de occidente, más conocida como la guerra entre esmeralderos, hechos que han dejado huellas muy marcadas en la vida de los boyacenses. Las secuelas son apreciables en el comportamiento violento de muchas personas y en las diversas violaciones a los derechos humanos e infracciones a las normas, como lo muestra un informe del Departamento de Policía de Boyacá, referenciado en el Plan de Desarrollo del departamento de Boyacá 2012-2015, donde se afirma: "los delitos de mayor impacto en el departamento son: las lesiones personales con un $46 \%$, la violencia intrafamiliar con un $39 \%$, el abuso sexual con 38\% y el suicidio con un 3\%, las ciudades donde más se presentan son Tunja, Sogamoso, Duitama y Chiquinquirá" (Gobernación 
de Boyacá, s.f., p. 20). Tales delitos son indicadores de las diversas situaciones de violencia y, por consiguiente, de una problemática relevante en contra de la convivencia pacífica.

El departamento está dividido en trece subregiones, una de ellas la subregión de occidente, con su capital de provincia: Chiquinquirá. Esta ha sido fuertemente influenciada por las dinámicas de violencia presentes en varios de los municipios que la conforman, originados principalmente por la explotación y comercio de las esmeraldas. Los conflictos en gran parte se resuelven de forma violenta, llegando incluso al uso de armas de fuego para silenciar a los opositores. Además, es un territorio donde la violencia se asocia a una cultura con ideario predominantemente machista, en el que impera la ley del más fuerte para ejercer dominio sobre otros, donde conseguir dinero por cualquier medio es habitual; es visible, además, la preferencia por la música que hace apología a la violencia, a diversas formas de trabajo ilícito, a las peleas de gallos y las apuestas. Estas situaciones tradicionalmente originan apasionamiento, crimen, intimidación, terror y desplazamiento, y se traducen en un modo de vida propio de la región, con incidencia directa en el municipio de Chiquinquirá, a cuya zona urbana continuamente arriban personas provenientes tanto de los municipios de la región como de otras poblaciones vecinas del norte de Cundinamarca (Simijaca, Susa y Fúquene) y sur de Santander, que por variadas razones, prioritariamente por la violencia, se asientan en los barrios periféricos trayendo consigo los conflictos y los deseos de retaliaciones, que no pocas veces terminan en asesinatos. Refiriéndose a esta problemática, el periodista y escritor Raúl Ospina Ospina (2016) señala:

La identidad cultural que tuvo Chiquinquirá hace unos cien años (la ciudad de tertulias, de los cien pianos, de los museos, de las retretas de la banda, de las serenatas con música de cuerda, de la poesía) se ha perdido en alto porcentaje e incluso tiende a desaparecer debido a un proceso de aculturación que se da por la presencia de gente de otras regiones, especialmente del occidente bajo, quienes llegan a habitar en su mayoría los barrios del suroccidente del municipio, entre ellos el Boyacá Alto y Boyacá Bajo. (Entrevista personal realizada por Ninfa Pinilla, 2016)

Su punto de vista pone de relieve los cambios que se han sucedido en la ciudad y que de algún modo se han originado por la llegada de un grueso de la población que busca mejores condiciones de vida o por situación de desplazamiento que, según la base de datos de la Consultoría Para los Derechos Humanos y el Desplazamiento (CODHES), es de 992 personas desde el año 1999 a 2012. La concejala Rosalba Burgos lo expresa en los siguientes términos: "la población de los barrios suburbanos Boyacá Alto y Boyacá Bajo derivan de una mezcla de culturas; en la mayoría de los casos no tienen una identidad con el contexto ni una cultura de pertenencia debido a su constante movilidad por su situación socioeconómica" (entrevista personal realizada por Ninfa Pinilla, 2016). 
Esta se convierte en una población flotante cuyos ingresos provienen de la labor que desarrollan en talleres de mecánica, en el reciclaje, en el servicio doméstico, como conductores, vendedores ambulantes, minoristas en la plaza de mercado, el comercio informal o simplemente no tienen ningún ingreso, por lo cual hay quienes se dedican al delito asociado a la irregularidad, lo que incrementa la problemática de inseguridad y de violaciones al orden público.

Dicha situación genera en muchas familias la incapacidad para garantizar a sus hijos e hijas las condiciones mínimas de supervivencia, la escolaridad y el tiempo para contribuir a una formación adecuada. Comúnmente, padres y madres deben salir desde muy temprano a cumplir con largas jornadas de trabajo informal, dejando los pequeños al cuidado de terceras personas e incluso solos. Consecuentemente, los niños y las niñas están permanentemente descuidados, duran mucho tiempo en la calle en compañía de personas que no les aportan modelos adecuados de comportamiento y están expuestos a situaciones de violencia que se añaden a la que muchas veces vivencian en el hogar. Estos factores, asociados a las precariedades propias del contexto, fortalecen las conductas de violencia que luego se traspasan al escenario de la escuela.

Frente a la problemática descrita, la participación del Estado y las políticas sociales son disímiles: mientras se hace presente con algunos subsidios, permanece ausente en la satisfacción de servicios públicos de calidad y en proyectos de desarrollo de las comunidades. En materia de organización comunitaria, los bajos índices lo dicen todo: la participación e iniciativa en proyectos que lleven a mejorar las condiciones de vida de los habitantes es mínima, por lo que prevalecen las formas individualistas en la solución de problemas y necesidades. De ahí que, en los últimos años, en estas zonas suburbanas de la ciudad se vive un aumento significativo de drogadicción, prostitución (especialmente infantil), tabaquismo, alcoholismo y abuso sexual. El consumo regular de alcohol lleva en muchos casos a que las personas pierdan el control de sus actos, se vuelvan más agresivas y lleguen hasta maltratar a su pareja, a sus hijos, a amigos y parientes o simplemente a generar conflicto con quienes se encuentren a su alrededor. Todo esto altera la sana convivencia, tanto de los vecinos como de la familia. Estos episodios son escuchados, vistos y aprendidos por los niños y niñas.

En muchos casos se vivencia la violencia intrafamiliar, sea entre las parejas o de padres a hijos, que para el año 2010 llegó a ser de 3.2 casos por cada 1.000 habitantes (Forensis, 2011) y para el año 2011 de 3.4 por cada 1.000 (Forensis, 2012) para ejercer la autoridad y lograr que el agredido haga o cumpla la voluntad de su agresor. Algunos padres de familia, en su afán de corregir a los hijos por las equivocaciones que cometen o por las cosas que dejan de hacer, imponen castigos físicos y verbales, lo cual aumenta el riesgo de permanencia en el ciclo de la violencia, tal como lo sugiere Chaux (2013, p. 44): “los mismos que cuando niños fueron expuestos a mucha violencia pueden terminar contribuyendo a que ésta se mantenga a largo plazo".

Por lo anterior se hace necesario fortalecer en la formación basada en la responsabilidad, la tolerancia, el respeto a sí mismos y hacia el otro con el fin de 
disminuir el riesgo relacionado con lo que expresa Farrington (1993, citado en Chaux, 2012, p. 44) cuando expresa que "si no se hace ninguna intervención, quienes son más agresivos en la niñez tienen una mayor probabilidad de ser los más agresivos en la edad adulta". De esta forma, la cadena interminable de conflictos y agresiones físicas o verbales a las que están sometidos los pequeños al interior de su familia, la escuela y en la calle les impide establecer relaciones armónicas y los puede llevar a tomar decisiones que repliquen o fortalezcan el ciclo de la violencia.

\section{Metodología}

En la sistematización de la experiencia pedagógica desarrollada en la Institución Educativa Liceo Nacional José Joaquín Casas, sede General Santander, del municipio de Chiquinquirá, seguimos un enfoque cualitativo, que desde la perspectiva de Creswell (1998, p. 15, citado en Vasilachis, 2006, p. 2) “es un proceso interpretativo de indagación basado en distintas tradiciones metodológicas que examina un problema humano o social" (). Interpretación que en este caso se hace para tomar como referente un entorno escolar específico, donde niñas y niños se vincularon a un proceso de mejoramiento de la convivencia escolar.

Para recopilar la información que permitiera reconstruir los aspectos más relevantes de la experiencia, acudimos a entrevistas semiestructuradas, por tener estas cierto grado de flexibilidad (Bernal, 2010, p. 257) y permitir una conversación donde pueden surgir nuevos cuestionamientos e información que no se habían contemplado en un comienzo. Estas fueron aplicadas a diferentes actores de la comunidad educativa y a personas que participaron en la experiencia. De manera alterna se llevó a cabo una revisión de documentos facilitados por la coordinación de la sede escolar, en los que se destaca una encuesta diagnóstica aplicada a los estudiantes de los grados tercero, cuarto y quinto de la sede en el año 2010, el proyecto Pedagogía de la Confianza donde se planean y ejecutan estrategias concretas y pertinentes para generar espacios de confianza, buen trato entre todos los miembros de la comunidad educativa y espacios apropiados para la formación personal y el ejercicio de los Derechos Humanos. También se acudió al uso de narrativas con siete estudiantes y la aplicación de una encuesta a setenta y un estudiantes que actualmente se encuentran cursando el grado undécimo y que participaron en la experiencia Pedagogía de la Confianza en el año 2011, cuando cursaban el grado quinto, en la sede General Santander, para conocer su concepción acerca del conflicto e incidencia en sus vidas.

Con la información obtenida de las entrevistas y la revisión documental se diligenciaron las matrices propuestas por el Colectivo de Educación para la Paz, que habían sido entregadas previamente. En un primer momento se respondió la guía de caracterización de la práctica pedagógica, que estaba divida en cuatro categorías: actores, relaciones, historia y práctica pedagógica. Posteriormente se utilizaron cuatro instrumentos: 1. Identificación y descripción de la experiencia; 
2. Historia y contexto de la experiencia; 3 . Actores vinculados a la experiencia; y 4. Relaciones entre actores vinculados a la propuesta, los cuales permitieron profundizar en los diferentes aspectos y elaborar el informe de sistematización de experiencias pedagógicas en la Guía 1.

Los estudiantes de grado décimo participaron comentando sus vivencias y narrando los aprendizajes y saberes que les aportó el proyecto para su vida. Así, la sistematización cumplió el propósito de desentrañar las especificidades de una experiencia pedagógica orientada a promover la convivencia escolar como hecho de paz y respeto a los derechos humanos.

\section{Experiencia pedagógica como foco de problematización}

La institución educativa Liceo Nacional José Joaquín Casas, sede General Santander, se encuentra ubicada en el barrio Ricaurte del municipio de Chiquinquirá, en cercanía a los barrios Boyacá Alto y Boyacá Bajo, los cuales le aportan la mayor cantidad de estudiantes con las características socioculturales anteriormente descritas. Por ello, no resultaba extraño que hacia los años 2009 y 2010, según la coordinadora de la sede, Angélica Rojas, el ambiente escolar se viera deteriorado por constantes conflictos que terminaban en agresiones verbales y físicas, incluso en peleas entre los estudiantes, especialmente en las horas de descanso. Tal como lo muestra una encuesta diagnóstica aplicada en la sede General Santander a los niños y niñas de los grados tercero, cuarto y quinto de educación básica primaria, con edades entre ocho y doce años, se evidenció que el 30\% de los estudiantes afirmaban haber vivido situaciones de conflicto y que en dichas situaciones el 77\% de ellos utilizó la agresión física y el 100\% agresión verbal (I. E. Liceo Nacional, 2010). Entre las formas más comunes de agresión se encontraban el uso de apodos denigrantes, expresiones verbales groseras, propinarse puños y patadas, escribir en las paredes mensajes amenazantes, entre otras acciones que generaban violencia escolar.

Ante tales hechos, los directivos y docentes recurrieron a los llamados de atención y a la aplicación de correctivos para, por lo menos, tratar de contener las manifestaciones de agresividad. Entre las acciones correctivas más comunes estaba no permitirles salir al descanso como los demás compañeros y llamar a los padres de familia para que asumieran responsabilidades, pero esta última acción muchas veces no tenía eco porque existían niños y niñas que no vivían con sus padres, sino con un familiar (tíos, abuelos, primos, etc.) que no deseaba recibir llamados de atención por el comportamiento de su acudido ni tenía interés en su formación. Con el tiempo, los procesos comenzaron a hacerse extensos, tediosos y las acciones inoperantes, pues no se lograba que el estudiante mejorara en sus relaciones interpersonales con los compañeros y demás miembros de la comunidad educativa. Por estos motivos se hizo necesario buscar estrategias pedagógicas que coadyuvarán con el mejoramiento del ambiente escolar. 
Fue así como surgió la estrategia Pedagogía de la Confianza, iniciativa enfocada a disminuir los altos niveles de agresividad que se estaban evidenciando entre los estudiantes. La experiencia se propuso, mediante la planeación y ejecución de estrategias pedagógicas y lúdicas, lograr la disminución de los constantes casos de agresiones verbales y físicas que se presentaban entre los educandos, sensibilizando a los niños y las niñas en la importancia del diálogo para tener espacios de esparcimiento en paz.

El proyecto se ejecutó durante los años 2011, 2012 y 2013. Las estrategias lúdicopedagógicas, para educar en un ambiente de confianza y respeto, contemplaron la realización de talleres relacionados con el buen trato, la convivencia, el modo cordial en las formas rutinarias de relacionarse consigo mismo y con los otros, valores éticos, derechos humanos, entre otras. Las actividades realizadas se describen con detalle a continuación:

- Club de gestores de paz: inicialmente, en cada salón se nombró un gestor de paz, pero luego se vio la necesidad de elegir a dos (un niño y una niña) mediante voto popular, teniendo en cuenta su liderazgo, fluidez verbal, buen trato con sus compañeros y espíritu conciliador. Se citaban a una reunión y se nombraba un secretario encargado de llevar las actas de las reuniones, se les explicaba el cargo que iban a desempeñar y funciones que tenían, especialmente si se producían situaciones de conflicto durante el descanso de los estudiantes; entonces, debían llamar a las partes y dialogar con ellos sobre los hechos acaecidos para llegar a un acuerdo. La constitución del Club se realizaba anualmente, en un acto protocolario donde se les hacía entrega de un carnet que los identificaba como gestores de paz, se les recordaban las funciones y se le explicaba a la comunidad el papel que tendrían estos niños en la solución de los conflictos que se presentaran entre estudiantes. A medida que los designados iban realizando su función, los compañeros aprendían a respetarlos, a hacerles caso y a atender sus sugerencias, especialmente si la cumplían bien.

- Mural del señor Cortesía: consistió en un cartel con frases o palabras alusivas al buen trato (imagen 1) para que a través del tiempo los niños y las niñas las interiorizaran. Además, cuando algún estudiante agredía a otro o decía alguna grosería, dentro de las acciones que se realizaban era ir al "mural del señor Cortesía" e invitarlo a buscar la frase que correspondía a ese momento. Con el tiempo, el mural se transformó en "Somos comunidad cortesía". 


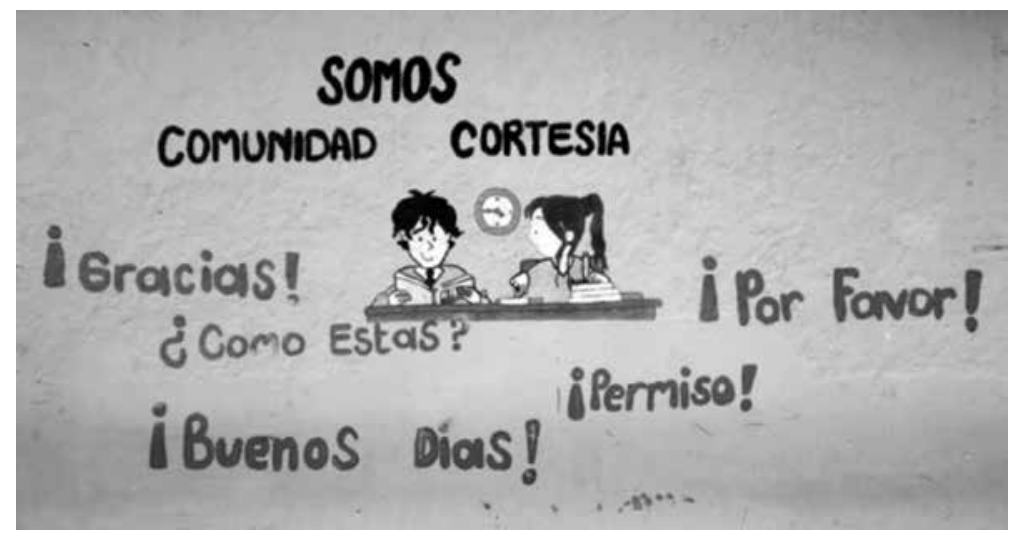

Fuente: archivo de la sede General Santander.

- Actividades deportivas: contemplaron partidos de fútbol y de baloncesto entre cursos, en los que el equipo ganador se definía no por el número de goles o de cestas, sino por haber cometido el menor número de faltas. El propósito era promover el juego limpio y dejar en los estudiantes el mensaje de que no todo se vale para poder ganar.

- Recitales de poemas, dramatizados y canciones con temáticas relacionadas con el buen trato y la paz: en el marco de esta actividad se presentaron poemas y canciones que promovían el crecimiento personal, el respeto hacia el otro, la amistad, la valoración de sí mismo y del otro, como el poema Desiderata, de Max Ehrmann.

- Urna del compromiso: se utilizó de la siguiente manera: cuando un estudiante generaba inconvenientes o situaciones que afectaban el ambiente escolar o la convivencia, debía escribir en un papel de color un compromiso de mejoramiento y depositarlo en la urna. Cada cierto tiempo se destapaba la urna y se leían nuevamente los compromisos para verificar que quienes los habían escrito hubiesen cumplido su promesa de mejoramiento.

- Izada de la bandera de la paz: dentro de las izadas de bandera del año escolar se programó una con la temática de la paz, ocasión en la que se izaba una bandera blanca como símbolo de que la institución constituía una comunidad en paz y tolerante.

\section{Resultados}

Después de tres años de aplicación del proyecto, según la coordinación institucional, mejoró el clima escolar: se empezaron a vislumbrar poco a poco cambios positivos en la población estudiantil, como la disminución de las peleas y 
agresiones tanto en las horas de descanso como en las aulas de clase e incluso a la salida de la jornada escolar; también se notó que los estudiantes comenzaron a solucionar los conflictos por sí mismos con el acompañamiento de los gestores de paz, reduciendo acciones represivas o sancionatorias propias de la autoridad de docentes o instancias institucionales.

Se logró involucrar a estudiantes que, a pesar de su corta su edad, asumieron con responsabilidad el rol de mediadores en situaciones complejas que se presentaban entre compañeros y que requerían de sus conocimientos, vivencias y propuestas para ser solucionadas de forma pacífica. Los gestores de paz se convirtieron en un apoyo importante para los docentes en cuanto al mejoramiento del ambiente escolar y la disminución de los niveles de agresividad. El 56\% de los estudiantes encuestados encuentran que la intervención de una tercera persona es favorable para solucionar los conflictos que se presentan entre las personas porque, según afirman ellos, “ayuda a calmar los ánimos, tener otros puntos de vista, ayuda a entrar en razón y entender al otro, fomenta el dialogo...". De otra parte, tal como lo comentan en sus relatos los estudiantes: "para mi punto de vista esto ayuda bastante, ya que cada gestor de paz podía entablar diálogos con los compañeros y buscar solución a sus problemas..."1, los gestores de paz coadyuvan a la resignificación de la convivencia escolar y la resolución pacífica de conflictos.

Los estudiantes adquirieron conciencia de que los conflictos se resuelven por medio del diálogo y que la violencia no genera ninguna solución; aprendieron a ser tolerantes, a respetar al otro y a aceptarlo como es; lograron mayor conocimiento de sí mismos, a mejorar su autoestima y a descubrir cualidades y habilidades que tal vez no creían poseer, tal como lo evidencian los relatos de estudiantes del grado décimo durante el año 2016, entre los que se reseña: "sí le queda a uno como una herramienta o una opción más civilizada para arreglar los conflictos y problemas que muchas veces se presentan dentro del plantel educativo y la adolescencia"2, y: "este proyecto me ayudó a mejorar como persona, ya que me di cuenta de que los conflictos se arreglan hablando, no peleando física y verbalmente..." ${ }^{3}$.

Estudiantes a quienes por múltiples problemáticas les cancelaron la matrícula en otras instituciones educativas, y que no los recibían prácticamente en ningún otro establecimiento, fueron integrándose a las dinámicas del proyecto y progresivamente construyeron mejores relaciones con sus compañeros y con el entorno, asegurando la permanencia y continuidad en el sistema educativo. Hoy varios de ellos están cursando su secundaria.

1 Base de datos de las autoras. Relato de una estudiante del grado décimo, participante de la experiencia en 2016.

2 Base de datos de las autoras. Relato de una niña de grado décimo, quien participó en la experiencia Pedagogía de la Confianza En el año 2016.

3 Base de datos de las autoras. Relato de un niño de grado décimo, quien participó en la experiencia Pedagogía de la Confianza En el año 2016. 
Entre los directivos docentes y docentes la experiencia pedagógica presentada permitió un mejor conocimiento de sus estudiantes y sus potencialidades, y el aprovechamiento de estas para mejorar el ambiente escolar, tan importante para el desarrollo del quehacer educativo y de la función de estos como formadores de seres humanos integrales.

Según afirmaciones de la coordinadora de la sede, no hubo necesidad de llevar casos al Comité de Convivencia Escolar para ser estudiados y buscar soluciones que muchas veces terminan en la sugerencia de cambio de institución.

La experiencia pedagógica incidió en las familias, puesto que varios niños y niñas de manera espontánea y con agrado comentaban a sus docentes el cambio que tuvieron con sus padres y de estos con ellos, reflejado en el trato afectuoso y de respeto, tal como lo expresan varios docentes entrevistados y la coordinadora Angélica Rojas.

Otro valor resultante fue la cooperación, que se consolidó en la medida en que los gestores de paz estuvieron dispuestos a ayudar a sus compañeros en la solución de los conflictos, desprendiéndose de tiempo que debieron quitarle al descanso y a otras actividades.

De igual manera, docentes, directivos docentes y ocasionalmente algunas entidades del orden municipal, como el Hospital Regional, la Policía de Infancia y Adolescencia y un padrino del proyecto, aunaron conocimientos, experiencias y saberes en la búsqueda de un mismo propósito: el mejoramiento del clima escolar a través de la resolución pacífica del conflicto.

\section{Conclusiones}

Teniendo en cuenta los resultados obtenidos, y para responder a los objetivos de la sistematización de la experiencia, se concluye:

La integración de la Pedagogía de la Confianza al Proyecto Educativo Institucional mejora la convivencia entre estudiantes e influye de manera positiva en la generación de ambientes escolares de paz favorables para el desarrollo de los aprendizajes y las habilidades sociales.

Las estrategias que se adopten alcanzan mayor impacto cuando tienen en cuenta la participación de toda la comunidad educativa no solo como receptora, sino como actora, tal como sucedió con los gestores de paz, acción que la motiva a coadyuvar en la resolución de los conflictos.

Existen mecanismos distintos a los puramente correctivos y sancionatorios como la mediación, el "mural del señor Cortesía" y las actividades lúdico-pedagógicas para generar conciencia en las bondades de la resolución pacífica de los conflictos y el buen trato, en especial entre los niños y las niñas. 
Las narrativas movilizadas durante la investigación lograron reconstruir y dar cuenta de la experiencia Pedagogía de la Confianza realizada en la Institución Educativa Liceo Nacional José Joaquín Casas de Chiquinquirá, que se convierte en un saber rescatado para darlo a conocer en diferentes escenarios.

La tarea esencial de los padres, madres y docentes es fomentar en el hogar y en la escuela un ambiente de sana convivencia donde se practiquen acciones positivas que motiven a los niños y niñas a actuar de manera responsable, solidaria y tolerante, que los lleve a tener una vida en armonía con sus semejantes.

\section{$\mathbf{R}_{\text {eferencias }}$}

Acosta, A. (2004). Regulación de conflictos y sentimientos. Recuperado de http:// wdb.ugr.es/ eirene/wp-content/files/publicaciones/ColeccionEirene/eirene_manual/Regulacion_de_Conflictos_y_Sentimientos.pdf

Alzate, S. R. (1998). Análisis y resolución de conflictos: una perspectiva psicológica. Bilbao: UPV-EHU.

Bernal, C. (2010). Metodología de la investigación. Bogotá: Editorial Pearson.

Burgos, R. (2016). Entrevista realizada por Ninfa Pinilla, 2 de junio de 2016. Chiquinquirá.

Carvajal, A., Urrea, P., y Soto, M. (2012). La convivencia escolar en adolescentes de cinco municipios de sabana centro departamento de Cundinamarca - Colombia (Tesis de maestría). Universidad de la Sabana. Chía, Cundinamarca.

Chaux, E. (2012). Educación, convivencia y agresión escolar. Bogotá: Ediciones Uniandes.

Congreso de la República de Colombia. Ley 115 de febrero 8 de 1994. Ley General de Educación.

Congreso de la República de Colombia. Ley 1620 de 2013. Ley de Convivencia Escolar.

Consultoría Para los Derechos Humanos y el Desplazamiento (base de datos). Consulta hecha el 27 de marzo de 2017.

Deutsch, M. (2003). La resolución del conflicto: procesos constructivos y destructivos (1973). En B. R. Domínguez y D. S. García, Introducción a la teoría del conflicto en las organizaciones. Madrid: Universidad Rey Juan Carlos.

Departamento Administrativo Nacional de Estadística (2011). Encuesta de convivencia escolar y circunstancias que la afectan - ECECA, para Estudiantes de $5^{\circ}$ a $11^{\circ}$ de Bogotá. Recuperado de https://www.dane.gov.co/files/investigaciones/boletines/educacion/presentacion_ConvivenciaEscolar_2011.pdf 
Forensis (2011). Comportamiento de la violencia intrafamiliar Colombia 2011. Bogotá: Instituto Nacional de Medicina Legal y Ciencias Forenses.

Forensis (2012). Comportamiento de la violencia intrafamiliar Colombia 2011. Bogotá: Instituto Nacional de Medicina Legal y Ciencias Forenses.

Girard, K., y Koch, S. (1997). Resolución de conflictos en las escuelas. Manual para educadores. Buenos Aires: Ed. Granica S. A.

Gobernación de Boyacá (s. f.). Plan departamental de desarrollo 2008-2011 ¡Para seguir creciendo! Tunja: Gobernación de Boyacá.

Gobernación de Boyacá (s. f.). Plan departamental de desarrollo 2012-2015, “Boyacá se atreve". Tunja: Gobernación de Boyacá.

Hernández, W., y Suárez, C. (2013). Aprendizaje emocional y violencia escolar: identificación del estilo de confrontación relacionado con la asertividad en episodios de violencia escolar interpersonal (Tesis maestría). Tunja: Universidad Santo Tomás.

Institución Educativa Liceo Nacional José Joaquín Casas - sede General Santander. (2010). Encuesta diagnóstica de Convivencia escolar. Bogotá.

Herrera, K., Rico, R., y Cortés, O. (2014). El clima escolar como elemento fundamental de la convivencia en la escuela. Escenarios, 12(2), 7-18.

Hevia, R. (2006). Frente a la crisis de sentido, una pedagogía de la confianza. Revista PRELAC (2), 70-75.

Hevia, R. (2016). La escuela desde la pedagogía de la confianza. Revista Mensaje. Recuperado de http://www.mensaje.cl/sociedad/ la-escuela-desde-la-pedagoga-de-la-confianza

Huertas, J. A., y Montero, I. (2002). Interacción en el aula. Buenos Aires: Impresiones Sudamérica.

Lederach, J. P. (1997). Enredos, pleitos y problemas: una guía práctica para ayuda a resolver conflictos. Santafé de Bogotá - Guatemala: Clara-Semilla.

Ministerio de Educación Nacional (2004). Estándares básicos de competencias ciudadanas. Bogotá: MEN.

Ministerio de Educación Nacional (2008). Guía No. 34. Guía para el mejoramiento institucional. Bogotá: MEN.

Ospina Ospina, R. (2016). Entrevista realizada por Ninfa Pinilla, 13 de julio de 2016. Chiquinquirá. 
Patiño, D. S. (2011). La convivencia escolar como elemento fundante para una formación en ciudadanía (Tesis de maestría). Universidad de Córdoba SUE Caribe. Montería.

Ramírez-López, C., A. y Arcila R., W. (2014). Violencia, conflicto y agresividad en el escenario escolar. Educación y Educadores, 16(3), 411-429. Recuperado de http://educacionyeducadores.unisabana.edu.co

Subsecretaría de Asuntos para la Convivencia y Seguridad Ciudadana. (2006). Convivencia y seguridad en ámbitos escolares de Bogotá D.C. Recuperado de http://www.elespectador.com/files/d8f4461c07272f8357a73012230992f3.pdf

Vasilachis, I. (2006). Estrategias de investigación cualitativa. Barcelona: Gedisa Editorial.

Zurbano D., de C. J. (2008). Bases de una Educación para la Paz y la Convivencia. Navarra: Departamento de Educación y Cultura. Recuperado de http:// 213.0.8.18/portal/Educantabria

\section{Anexos}

\section{Guía 1}

Colectivo Educación para la Paz

Pilar investigativo

Propuesta de investigación colectiva del Colectivo Educación para la Paz

Protocolo de aplicación. Instrumento 1. Caraterización

Instrumento de caracterización de la práctica pedagógica:

El instrumento de caracterización tiene el propósito de ayudarle a organizar la información que pueda recolectar sobre la práctica pedagógica que pretende estudiar. El instrumento está divido en cuatro categorías: actores, relaciones, historia y práctica pedagógica. Se espera que, para cada una de ellas, usted aporte el mayor número de datos que le sean posibles, así como descripciones detalladas sobre lo que las personas involucradas en la práctica le relaten.

Categoría 1: actor

Objetivo: describir en detalle las personas y los grupos que configuran el contexto de la práctica que se pretende estudiar. 
A continuación se definen los tipos de actores que se relacionan en el instrumento de caracterización:

1. Profesores y directivos: profesores y/o directivos de la institución educativa que dirigen y coordinan la práctica.

2. Estudiantes: están directamente involucrados en el desarrollo de la práctica.

3. Grupos del contexto: personas, grupos, grupos religiosos, empresas, cooperativas, universidades, colegios, organismos internacionales u organizaciones no gubernamentales que, aunque no están vinculados directamente en la práctica, proveen soporte, asesoría, capacitación, recursos, personal o cualquier otra forma de apoyo al desarrollo de la práctica o, en su defecto, amenazan el desarrollo de la misma.

4. Instituciones gubernamentales: secretarías, ministerios, consejos regionales, agencias del gobierno local, regional y nacional, ministerio público.

Categoría 2: relaciones

Objetivo: describir en detalle las relaciones entre las personas y los grupos que configuran el contexto de la práctica que se pretende estudiar.

A continuación se formulan las preguntas a responder en el marco del instrumento:

1. ¿Qué relaciones hay entre los actores descritos anteriormente?

2. ¿Cómo son las relaciones de estos actores con la práctica?

\section{Categoría 3: historia}

Objetivo: describir en detalle las condiciones históricas relacionadas con el contexto de emergencia de la práctica que se pretende estudiar.

A continuación se formulan las preguntas a responder en el marco del instrumento:

1. ¿Cuál es la historia del lugar y de la institución en el que se desarrolla la práctica? 
2. ¿Qué problemáticas del contexto dieron lugar al diseño e implementación de la práctica pedagógica en su versión actual (prácticas en derechos humanos o en proceso de paz)?

3. ¿Qué factores/actores de la historia contribuyen al surgimiento de la práctica?

Categoría 4: Práctica pedagógica (en derechos humanos o en educación para la paz)

Objetivo: describir en detalle las características de la práctica que se pretende estudiar.

A continuación se formulan las preguntas a responder en el marco del instrumento:

1. ¿Cuáles son las razones por las cuales esta práctica nace?

2. ¿Cuáles eran los objetivos iniciales de la práctica cuando nació?

3. ¿Qué cosas han cambiado sobre los objetivos de la práctica?

4. ¿Cuáles son los actores clave vinculados al nacimiento de la práctica?

5. ¿Qué estrategias y actividades se desarrollan en el marco de esta práctica?

6. ¿Qué efectos ha tenido esta práctica con relación a el problema identificado, los actores, las relaciones entre ellos, las instituciones?

7. ¿Qué percepciones tienen los grupos del contexto con relación al desarrollo de esta práctica (positivas, negativas, de resistencia, de inconformismo, de amenaza, etc.)

8. ¿Cuáles son los aprendizajes más significativos que han tenido los actores -estudiantes, profesores, directivos, comunidad- a partir del desarrollo de esta práctica? 